\title{
Fear but not awareness predicts enhanced sensory processing in fear conditioning
}

\author{
STEPHAN MORATTI, ${ }^{a}$ ANDREAS KEIL, ${ }^{a}$ AND GREGORY A. MILLER ${ }^{b}$ \\ a Department of Psychology, University of Konstanz, Konstanz, Germany \\ ${ }^{b}$ Department of Psychology and Beckman Institute Biomedical Imaging Center, University of Illinois at Urbana-Champaign, Urbana, Illinois, USA
}

\begin{abstract}
It is not clear whether enhanced cortical processing of reinforced stimuli as reported by neuroimaging studies is due to expectancy of an aversive event alone or to activation of the fear system. The present study investigated cortical and autonomic responses of aware participants using an instructed fear conditioning design. Steady-state visual evoked fields (ssVEF) and heart rate change were recorded to assess sensory processing and activation of the fear system by reinforced $(\mathrm{CS}+)$ and nonreinforced stimuli $(\mathrm{CS}-$ ). Participants who showed heart rate acceleration demonstrated increased ssVEFs in visual and parietal cortex during CS + in acquisition trials. Heart rate decelerators did not show enhanced cortical activation with respect to the CS+. Participants in both groups reported awareness of CS-US contingencies. Awareness of stimulus contingency in fear conditioning seems not to be sufficient to elicit enhanced visual cortical processing.
\end{abstract}

Descriptors: Fear conditioning, Fear system, MEG, Heart rate, Steady-state visual evoked field potentials, Awareness

The extent to which higher order cognitive functions like attention and conscious awareness are a prerequisite for Pavlovian fear conditioning has been debated intensively (Lovibond \& Shanks, 2002; Wiens \& Öhman, 2002). However, many studies have demonstrated that Pavlovian fear conditioning may take place without conscious awareness of the CS-US relationship via backward masking of the CS+ (Esteves, Parra, Dimberg, \& Öhman, 1994; Morris, Öhman, \& Dolan, 1998; Olsson \& Phelps, 2004; Wiens, Katkin, \& Öhman, 2003) or via subthreshold presentation (Knight, Nguyen, \& Bandettini, 2003). Supporting evidence also comes from a study of a cortically blind patient (Hamm et al., 2003). These studies indicate that conscious attentional processes are not necessary for CS-US associations but these may be mediated by both explicit and implicit representations (Öhman \& Mineka, 2001). In a two-level account of human fear conditioning, fear-conditioned memories are considered implicit memories that can be activated by a fast direct thalamicamygdaloid route without conscious awareness of CS-US contingencies. In contrast, learning that a stimulus (or context) predicts a threatening event is considered an explicit memory process, which depends on conscious representations of the CSUS relationships (for an extensive review, see Hamm \& Weike, 2005).

We thank Leonie Koban and Ursula Lommen for help with data acquisition. Rescarch was supported by the Deutsche Forschungsgemeinschaft (DFG).

Address reprint requests to: Stephan Moratli, Department of Clinical Psychology, University of Konstanz, P.O. Box D25, D-78457 Konstanz, Germany. E-mail: Stephan.Moratti@uni-konstanz.de.
The amygdala has been demonstrated to be a core structure for Pavlovian fear conditioning (Davis, 1997; LeDoux, 2000; Shi \& Davis, 2001) and may mediate implicit learning via fast routes, potentially circumventing neocortex (Hamm et al., 2003; LeDoux, 1993). Thus, it probably represents an important element of a first-level fear memory system. However, cognitive theories of classical conditioning also highlight the role of higher order cognitive processes such as expectancy and attention (Pearce \& Hall, 1980; Rescorla, 1988), which involve neural activity in cortical networks, including deeper structures such as the hippocampus. These cortical structures and their functions may thus be closely related to the second level of the two-level account proposed by Hamm and Weike (2005). Besides elevated activation of the amygdala in response to the reinforced visual CS, that is, the CS+ (Büchel, Morris, Dolan, \& Friston, 1998; Cheng, Knight, Smith, Stein, \& Helmstetter, 2003; LaBar, Gatenby, Gore, LeDoux, \& Phelps, 1998), hemodynamic neuroimaging studies have demonstrated differential activation of frontal, temporal, parietal, visual cortices, and the anterior cingulate (Büchel et al., 1998; Cheng et al., 2003; Fischer, Andersson, Furmark, Wik, \& Fredrikson, 2002; Fredrikson, Wik, Fischer, \& Andersson, 1995; Hugdahl, 1998; Knight, Cheng, Smith, Stein, \& Helmstetter, 2004; LaBar et al., 1998), structures considered part of a general cortical attention system (Fernandez-Duque \& Posner, 2001). For instance, Armony and Dolan (2001) have demonstrated that a visual context stimulus predictive of the auditory aversive event modulated cortical activation for an auditory $\mathrm{CS}+$. This activation was accompanied by enhanced activity in parietal cortex, further illustrating the role of cortical attention networks in associating a context with contingencies. 
However, the extent to which the enhanced cortical processing of the $\mathrm{CS}+$ is due to expectancy alone or is mediated by activation of the fear system is not known.

Network theories of emotional processing emphasize the involvement of motive systems that drive attention toward stimuli of high biological significance, which has been labeled "motivated" or "natural selective attention" (Keil, Moratti, Sabatinelli, Bradley, \& Lang, 2005; Lang, Bradley, \& Cuthbert, 1997). Consistent with this view, it has been shown that highly arousing emotional visual stimuli are detected faster (Öhman, Flykt, \& Esteves, 2001), provoke longer exploration times (Bradley, Codispoti, Cuthbert, \& Lang, 2001), elicit autonomic reactions that are associated with attentional processes (Lang et al., 1997), and facilitate sensory processing in visual brain areas (Bradley et al., 2003; Keil et al., 2002; Schupp, Junghofer, Weike, \& Hamm, 2003). Although these approaches used complex natural scenes as stimuli, the concept of "motivated attention" as a reflexive reaction of an organism is of special interest for fear conditioning using simple visual stimuli. The concept of "motivated attention" represents a framework to differentiate between reflexive attentional processes driven by the motivational significance of a stimulus (e.g., threat) and volitional attentional processes driven by expectancy in fear conditioning.

Steady-state visual evoked responses have been intensively used to study the electrocortical responses underlying attentional processes during long-lasting visual stimulus presentation (Morgan, Hansen, \& Hillyard, 1996; Müller \& Hillyard, 2000; Müller \& Hübner, 2002). Further, the typical CS+ and CS presentation lasting several seconds is compatible with the steady-state visual evoked fields (ssVEF) paradigm, permitting the study of time-varying, reentrant modulation of visual processing in the course of ongoing stimulus viewing (Silberstein et al., 1990). In ssVEF designs, visual stimuli are typically presented repetitively, being modulated in luminance at a frequency of 6-8 Hz or greater for several seconds or more (Regan, 1989).

The sSVEF is the neuromagnetic counterpart of the steadystate visual evoked potential (ssVEP). It can be recorded with MEG as an ongoing oscillatory neuromagnetic response having the same fundamental frequency as the driving stimulus and can be localized in visual cortex (Müller, Teder, \& Hillyard, 1997). Steady-state visual evoked brain responses have been used to track the allocation of visual attention, with the electrocortical steady-state response being greater for attended than for unattended visual stimuli (Morgan et al., 1996; Müller \& Hillyard, 2000; Müller \& Hübner, 2002). This finding may reflect amplification of neural mass oscillations in the visual system (Hillyard et al., 1997).

Highly arousing affective stimuli generate greater ssVEPs/ ssVEFs than neutral pictures in visual and fronto-parietal networks, pointing to an involvement of higher order attentional mechanism in processing of pictures bearing high motivational relevance (Kemp, Gray, Eide, Silberstein, \& Nathan, 2002; Moratti, Keil, \& Stolarova, 2004). Recently, Keil and coworkers (2005) have shown an additive enhancement of the ssVEP amplitude by visual spatial attention and by the motivational relevance of a stimulus, demonstrating a functional overlap of neural systems processing high arousing stimuli and mediating spatial visual attention. Taken together, ssVEPs/ssVEFs may not only reflect stimulus-driven neural oscillations in early visual cortex but also in higher order cortical networks, possibly exerting top-down influences (for examples of localized ssVEP/ssVEF generators in higher order networks, see Moratti et al. 2004; Perlstein et al., 2003).

Moratti and Keil (2005) conducted a fear conditioning study using a ssVEF design to study reentrant modulation of visual processing in the time course of ongoing $\mathrm{CS}+$ and $\mathrm{CS}-$ viewing. Only participants exhibiting relative heart rate acceleration in response to the $\mathrm{CS}+$ showed facilitated visual processing of the $\mathrm{CS}+$ after learning trials. Relative heart rate acceleration following initial heart rate deceleration was interpreted as a marker for the presence of fear when viewing the CS + (cf. Hodes, Cook, \& Lang, 1985). Most participants (14 out of 17) could not report CS-US contingencies at the end of the experiment, demonstrating that facilitated CS+ processing in parieto-occipital cortex did not depend on contingency awareness. However, that study was not designed to evaluate CS-US contingency awareness. In addition, possible interactions of awareness and fear responses could not be followed using group comparisons given the low number of aware participants.

Given that a growing body of evidence indicates that awareness of stimulus contingencies is not necessary for fear conditioning (see above), the present study examined whether expectancy and awareness of stimulus contingency are sufficient for enhanced visual $\mathrm{CS}+$ processing in cortical sensory and attention networks or whether this process depends on the fear response evoked by the CS+. To overcome the shortcomings with respect of the evaluation of CS-US contingency awareness of the previous fear conditioning study using a ssVEF design (Moratti \& Keil, 2005), we conducted the present study controlling for contingency awareness by employing an instructed fear conditioning paradigm.

CS-US contingency awareness was established (1) by explaining to the participants that the $\mathrm{CS}+$ would predict an aversive event and (2) by using a $100 \%$ reinforcement schedule. Awareness was assessed using a recognition task at the end of the experiment. To determine whether awareness of the CS $+/ \mathrm{US}$ relationship alone elicits enhanced cortical processing of the visual CS+ (a "to-be-attended" effect driven by expectancy), a measure of the fear state was necessary. Work on autonomic fear conditioning has demonstrated that not all subjects acquire a fear response during fear conditioning as indexed by heart rate acceleration and that some participants show heart rate deceleration to the CS + instead (Hamm \& Vaitl, 1996; Hare \& Blevings, 1975; Hodes et al., 1985). For example, Hamm and Vaitl (1996) demonstrated that only heart rate accelerators exhibited potentiated startle responses, a measure of activation of the defense system (Lipp, Sheridan, \& Siddle, 1994), whereas skin conductance was elevated for the $\mathrm{CS}+$ for both groups. Therefore, it has been hypothesized that heart rate accelerators acquire a fear response, whereas heart rate decelerators merely learn that the CS+ signals another event (Hamm \& Vaitl, 1996; Hodes et al., 1985). Thus, considering heart rate responses to the CS+ could clarify whether the CS + signaling of an aversive event is or is not a sufficient prerequisite to activate the defense system. Therefore, heart rate changes to the $\mathrm{CS}+$ and $\mathrm{CS}-$ were recorded to measure activation of the fear system during the present fear conditioning procedure

In the present study, the CS + and CS - were presented in a flickering mode, to examine the ssVEF during and after learning. We hypothesized that if a previously neutral stimulus gains motivational relevance by fear conditioning, $\mathrm{CS}+$ processing should be facilitated compared to the unreinforced $\mathrm{CS}-$. This in turn was expected to be reflected in enhanced ssVEF amplitude in 
visual sensory and attentional cortical networks as reported in previous work using affective picture stimuli or stimuli associated with an aversive event (e.g., Moratti et al., 2004; Moratti \& Keil, 2005).

It is not clear, however, whether such activity modulation would be due to expectancy in fully contingency-aware subjects or, alternatively, by motivated attention driven by threat. Because heart rate acceleration versus deceleration during fear conditioning seems to indicate the presence and absence of fear system activation (Hamm \& Vaitl, 1996; Hare \& Blevings, 1975; Hodes et al., 1985), two competing predictions can be made for $\mathrm{CS}+$ facilitation in visual cortex. First, if an enhanced ssVEF amplitude in cortical sensory and attention networks is only a tobe-attended effect because of expectancy induced by the contingency awareness, all participants should show enhanced ssVEFs to the $\mathrm{CS}+$, regardless of whether their heart rate accelerates or decelerates to the CS+ during acquisition. Alternatively, if enhanced ssVEF amplitude is due to motivated attention directed by a fear-relevant stimulus, only heart rate accelerators should exhibit greater ssVEFs to the CS + compared to the CS - . The former result would indicate that volitional attention processes such as expectancy of an aversive event are sufficient to elicit facilitation of visual $\mathrm{CS}+$ processing. The latter result would lead to the conclusion that the activation of the defense system is necessary and that expectancy alone is not sufficient to produce facilitation of $\mathrm{CS}+$ processing in visual cortex.

\section{Methods}

\section{Participants}

Twenty healthy subjects ( 10 women, 10 men) participated in the study after having given written informed consent. Two of the 20 subjects were left-handed, and 18 were right-handed according to a standard handedness inventory (Oldfield, 1971). The mean age of the sample was 25.7 years (range: 19-55 years). They had normal or corrected-to-normal vision and no family history of photic epilepsy. The study was approved by the local ethics committee.

\section{Stimuli and Procedure}

Two gray-shaded $45^{\circ}$ gratings $(0.31$ cycles $/ \mathrm{cm}$, square wave) oriented perpendicular to each other served as conditioned stimuli. One of the two gratings served as the CS+ or CS - counterbalanced across subjects. Visual stimuli were projected on a screen in a magnetically shielded $M E G$ chamber using a video projector (JVC DLA-G $11 \mathrm{E}$ ) and a mirror system. Visual stimuli subtended a visual angle of $8^{\circ}$ both horizontally and vertically and were presented centrally. The unconditioned stimulus (US) consisted of a 95-dB SPL white noise with instantaneous onset delivered binaurally by an air tube system attached to a sound amplifier (BTi ASG, 1996)

The delay fear conditioning paradigm consisted of habituation, acquisition, and extinction trial blocks coupled with a visual discrimination task. A fixation cross of $2 \mathrm{~cm} \times 2 \mathrm{~cm}$ appeared in the middle of the screen throughout the experiment during $\mathrm{CS}+$ and $\mathrm{CS}-$ presentation and intertrial time periods. In each trial block, $20 \mathrm{CS}+, 20 \mathrm{CS}-$, and 6 target trials were shown. On each trial, the grating was presented for 5000 ms with a sinusoidally modulated luminance variation at $12.5 \mathrm{~Hz}$. The intertrial interval varied randomly between 6 and $8 \mathrm{~s}$. As ssVEPs/ssVEFs are highly sensitive to fluctuations in vigilance, arousal, and attention, we introduced a simple target detection task aiming to motivate subjects to maintain an alert and attentive state throughout the experiment. On the six target trials, the red fixation cross turned into blue for $500 \mathrm{~ms}$ beginning at $1000 \mathrm{~ms}$, $2500 \mathrm{~ms}$, or $4000 \mathrm{~ms}$ after CS + or CS - onset (three CS + and three CS - target trials), each lag being equally probable. Subjects were instructed to press a button with their right index finger as soon as they detected the color change of the fixation cross. The CS+, CS - , and target trials were presented in random order. During the habituation and extinction blocks, the US was not presented. In acquisition trials, the US was presented during the last second of CS + presentation and terminated at CS+ offset.

The experimental session began with instructions on the screen explaining the task for target trials and assuring subjects that no loud noise would be delivered during the habituation block. Subjects were asked to fixate the central cross throughout the experiment. A I-min break followed the habituation block, and participants were instructed that during the next block one of the two grating patterns would predict a loud noise " One of the two gratings that will be shown to you will predict the loud noise. Please pay attention to which of the two gratings predicts the noise, as we will ask you about this at the end of the experiment."). The extinction block started without a break after the acquisition trials and without informing subjects that the US would no longer occur. After the experiment, participants were shown the patterns that served as CS + and CS - and were asked to identify the pattern that predicted the loud noise. Participants correctly recognizing the $\mathrm{CS}+$ were scored as aware of the $\mathrm{CS}$ US contingency. After the experiment subjects were paid $15 E$ (approximately US\$15) for participation.

\section{Data Acquisition and Reduction}

The MEG was recorded continuously and digitized at a rate of $254.3 \mathrm{~Hz}$, using a 148-channel whole-head system (Magnes 2500 WH, 4D Neuroimage, San Diego, CA). A bandpass filter of $0.1-50 \mathrm{~Hz}$ was applied online. The electroocculogram and the electrocardiogram acquisition was done with a Synamps amplifier (Neuroscan, El Paso, TX) using $\mathrm{Ag} / \mathrm{AgCl}$ electrodes and with the same sampling rate and online filter as the $M E G$ recording. Two electroocculogram electrodes were placed near the left and right outer canthi and two above and below the right eye. Two electrocardiogram electrodes were placed at the left lower costal arch and the right collarbone. An electrode at the right mastoid served as ground. The head shapes of the subjects were digitized, and index points (left and right periauricular points, nasion, and two additional locations at the forehead) were determined to calculate head position within the MEG helmet for source analysis.

The ssVEFs were derived separately for each condition (CS+, $\mathrm{CS}-$ ) and each trial block (habituation, acquisition, extinction) by averaging each channel of MEG data for $4500 \mathrm{~ms}$ beginning $500 \mathrm{~ms}$ prior to CS onset. The 500 -ms prestimulus baseline was subtracted from the average. The last $1000 \mathrm{~ms}$ of CS presentation were not analyzed (resulting in a $4000 \mathrm{~ms}$ poststimulus time window) because the US could have startled the subject and produced movement artifacts. Target trials were not analyzed. Eye artifacts were corrected using the algorithm implemented in BESA software (Berg \& Scherg, 1994). Additionally, all data were inspected visually for movement artifacts.

A subspace out-projection (Uusitalo \& Ilmoniemi, 1997) of the eye artifact topography was applied to the ssVEFs to account 
for topography distortions introduced by the eye correction algorithm. Subsequently, the ssVEF amplitude was estimated by means of complex demodulation (Regan, 1989) at the stimulation frequency of $12.5 \mathrm{~Hz}$ to obtain stimulus-driven sine and cosine parts of the demodulated SSVEFs over time. To this end, data were multiplied with $12.5 \mathrm{~Hz}$ sine and cosine functions and a Butterworth zero-phase (order of 10) low-pass filter of $1 \mathrm{~Hz}$ was applied to the resulting time series before obtaining the vector length of the sine and cosine parts as a measure of time-varying amplitude. The narrow low-pass filter was chosen to obtain sufficient frequency resolution to separate the driving stimulus frequency from alpha background activity. Given the trade-off between time and frequency resolution, high frequency specificity resulted in relatively poor time resolution (see Results)

Before source estimation, a fast Fourier transformation (FFT) was calculated across the entire poststimulus ssVEF for each MEG channel, subject, and condition to verify if the number of epochs available produced a distinct power peak in the frequency spectrum at the $12.5-\mathrm{Hz}$ stimulation frequency. The minimum number of segments available per condition and block was 17. Mean power values were submitted to statistical analysis as described below.

A minimum norm estimation procedure described in detail elsewhere (Hauk, Keil, Elbert, \& Müller, 2002; Moratti et al., 2004) was applied to estimate the cortical origin of the ssVEFs. This procedure does not provide localization of structures in the sense of functional neuroanatomy, but aims to provide an estimate of generator structures at a lower spatial resolution. A spherical shell with 350 evenly distributed dipole locations served as the source space. The radius of the shell was $80 \%$ of the radius of the fitted sphere to the individuals' headshapes (Hauk et al., 2002).

The aforementioned subspace out-projection was also applied to the leadfield matrix that described the sensitivity of the sensors to the dipoles in the source space and contributed to the minimum norm solution. The regularization parameter $\lambda$ was derived from the averaged prestimulus baseline of each condition and trial block for each subject using the L-curve method (Hansen \& O'Leary, 1993). This was done to obtain an estimate of noise levels inherent in the data, as a basis for regularization. To avoid artifactual condition effects caused by different regularizations, the highest $\lambda$ value resulting from the $L$ curves for each subject was chosen for the minimum norm estimation.

Finally, the minimum norm estimation was calculated separately for the sine and cosine parts of the $12.5-\mathrm{Hz}$ demodulated signals for each condition, trial block, and subject. This procedure was derived from an algorithm calculating the minimum norm estimation in the frequency domain (Jensen \& Vanni, 2002). The square root of the sum of squares of the minimum norm amplitudes for the sine and cosine parts served as a measure of the total $12.5-\mathrm{Hz}$ current source density over stimulus presentation time:

$$
\begin{aligned}
A m p_{i}(t)= & \sqrt{A m p_{i, \text { sine }}^{2}(t)+A m p_{i . \text { cositu }}^{2}(t)}, i \in[1, \ldots, 350], \\
& t \in\left[0 m s_{,}, \ldots, 4000 m s\right]
\end{aligned}
$$

where $A m p,(t)$ is the total amplitude of the $12.5-\mathrm{Hz}$ demodulated ssVEF at dipole location $i$ and time $t$, and $A m p_{i, \text { sinc }}(t)$ and $A m p_{i, c o s i n c}(l)$ are the corresponding amplitudes of the sine and cosine parts for time $t$. The minimum norm amplitudes were averaged across three time windows $(\ell 1=0-1.3 \mathrm{~s} ; \iota 2=1.3-2.6 \mathrm{~s}$; $t 3=2.6-3.9$ s) for each condition ( $\mathrm{CS}+, \mathrm{CS}-$ ), trial block (habituation, acquisition, extinction), and subject. Finally, the minimum norm solutions were projected onto a schematic brain implemented in the Matlab package EMEGS (see www.unikonstanz.de/win/emegs; Junghöfer \& Peyk, 2004) and submitted to statistical analyses.

As with the MEG data, only the first $4000 \mathrm{~ms}$ of heart rate change during stimulus presentation were analyzed. Habituation and target trials were not analyzed. $R$-waves were detected in the digitized EKG, and interbeat intervals (cardiac time) were converted to beats per minute (real time) every $500 \mathrm{~ms}$. Heart rate during a 2000-ms prestimulus baseline was subtracted from the trial after averaging within CS type and subject.

\section{Statistical Analysis}

The mean $12.5 \mathrm{~Hz}$ power values of $\mathrm{CS}+$ and $\mathrm{CS}-$ conditions estimated across the entire poststimulus ssVEFs in the sensor space were submitted to paired $t$ tests for the acquisition and extinction blocks, respectively.

Within each block of acquisition and extinction, the interaction between condition (CS+, CS -$)$ and time $(t \mathrm{I}=0-1.3 \mathrm{~s}$; $t 2=1.3-2.6 \mathrm{~s} ; t 3=2.6-3.9 \mathrm{~s})$ was modeled at 273 of the 350 sources of the minimum norm estimation shell to identify source clusters of differential activations for the CS + and CS - . Sources at the bottom of the shell were discarded and not considered as meaningful because of their distance to the MEG sensors (reducing the number of dipoles from 350 to 273). The interactions were derived from a linear mixed-effects model applying restricted maximum likelihood estimation (REML) for parameter estimation. A detailed introduction to linear mixed-effects models in psychophysiological research can be found in Bagiella, Sloan, and Heitjan (2000), and the REML method is described in Searle, Casella, and McCulloch (1992).

To account for dependencies of observations over time, which are increased by the high frequency resolution and poor time resolution of the present complex demodulation, a first-order autoregressive covariance structure developed for time-series data (Pinheiro \& Bates, 2000) was applied in the linear mixedeffects model. The factors condition and time were treated as fixed effects. The intercept at the levels subject and condition within subject were treated as random effects. Finally, the $p$ values for the interaction terms were corrected for 273 multiple tests by the false discovery rate procedure (Benjamini \& Hochberg, 1995) and mapped onto the same schematic brain as the minimum norm solutions. Source clusters were considered as meaningful whenever at five or more neighboring source locations the interaction terms survived the false discovery rate procedure and thus consisted of sources with associated corrected $p$ values less than .05. To localize time windows of differentiated CS $+/ C S-$ activations, the average amplitude across significant source clusters within each time window and condition was submitted to paired $t$ tests, and $p$ values were Holm corrected (Holm, 1979). The entire statistical procedure was applied to heart rate accelerators and decelerators (see below) separately.

To identify heart rate accelerators and decelerators, a cluster analysis procedure described by Hodes et al. (1985) was applied to the heart rate change waveforms obtained during CS + depiction in acquisition trials. Heart rate change waveforms for heart rate accelerators and decelerators were averaged across three time windows $(\mathrm{tl}, \mathrm{t} 2, \mathrm{t} 3)$ for each condition $(\mathrm{CS}+, \mathrm{CS}-)$, the last two trial blocks (acquisition and extinction), and each subject. Because analyzing differences in heart rate time course can be more meaningful than simply comparing means 
(Wilson, 1974), the linear mixed-effects model described above was used, except that the factors time and time ${ }^{2}$ (three time windows allow polynomial fits up to $2^{\circ}$ ) served as ordered fixed effects to assess linear and quadratic trends. As heart rate waveforms can be characterized by triphasic patterns (Lang \& Hnatiow, 1962), a decelerative heart rate waveform would be reflected in a linear decrease in mean heart rate change values across the three time windows $(t 1, t 2, t 3)$. An accelerative pattern would be characterized by an inverted $U$-shaped time course across the three time intervals, reflecting heart rate acceleration in the second time slot.

Finally, a Pearson product moment correlation was calculated for each source, between the difference between the CS+ and $\mathrm{CS}$ - minimum norm amplitudes within the last time window (i.e., 13 as determined by statistical mapping of the Condition $x$ Time interaction and paired $t$ tests; see above) and the mean heart rate change values across the second time interval of stimulus presentation. According to a triphasic heart rate response model, the second time interval was chosen for the heart rate values to characterize subjects that accelerate or decelerate. The associated $p$ values were false discovery rate corrected, and the corresponding correlation coefficients were mapped in source space to visualize the relationship between heart rate change and oscillatory brain activity. Further, the mean difference between $\mathrm{CS}+$ and CS - activation within the last time window across statistically significant source clusters (more than five neighboring significant sources) emerging from the correlation analysis and the mean heart rate change values of the second time window (t2) were submitted to regression analysis.

To complement the regression analysis and to account for the observation that not all heart rate accelerators could push their heart rate above the zero baseline after an initial deceleration (see below), we entered the difference of the fastest heart rate value of the second and the slowest heart rate change of the first time window for each subject (the difference score will be referred to as Al) instead of the absolute heart rate change into the same regression analysis as decribed above.

\section{Results}

At the end of the session, all subjects correctly identified the grating that served as their $\mathrm{CS}+$ during acquisition.

\section{Heart Rate Data}

Cluster analysis separated two groups of participants showing different heart rate response patterns to the CS+ during acquisition trials. There were no significant differences in baseline heart rate between the two groups. Figure I depicts the heart rate waveforms of the two heart rate responder groups, accelerators $(N=9)$ and decelerators $(N=11)$.

Accelerators. A polynomial fit to the mean heart rate change values across three time intervals of CS presentation (see Methods) during acquisition indicated that heart rate accelerators responded to the $\mathrm{CS}+$ with a heart rate increase during the second time interval, whereas heart rate did not change during CS - presentation (quadratic time $\times$ condition: $F[1,32]=4.0$, $p=.055$; quadratic time during CS+: $F[1,[6]=7.1, p<.05$; quadratic time during CS - : n.s.; see Figure 1A). During extinction trials, heart rate accelerators showed a gradual, nonsignificant deceleration to both $\mathrm{CS}+$ and $\mathrm{CS}-$, with no interaction between a linear or quadratic trend and condition (Figure lB).

Decelerators. During acquisition, heart rate decelerators showed greater deceleration to the CS+ than to the CS - (interaction linear time $\times$ condition: $F[1,40]=6.3, p<.05$; linear time CS+: $f[1,20]=27.1, p<.0001$; linear time $C S-$ : $F[1,20]=5.2, p<.05$, see Figure $1 \mathrm{~A})$. In extinction trials, a

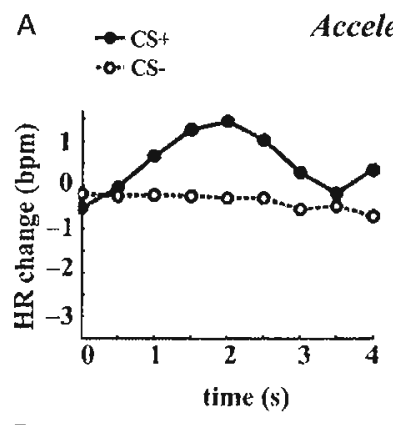

B

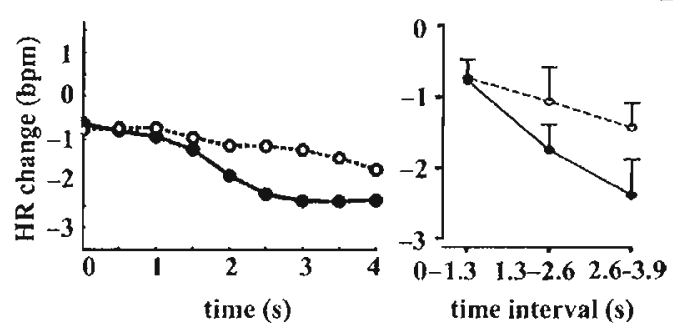

Acquisition

\section{Decelerators}

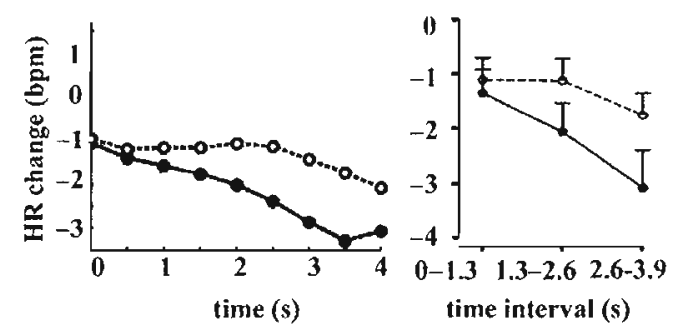

Extinction

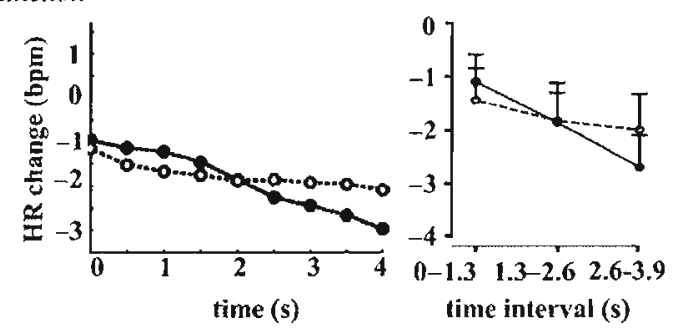

Figure 1. Hcart rate responses for heart ratc accelcrators and decclcrators during acquisilion (A) and cxtinction (B). The left panels of $\mathrm{A}$ and $\mathrm{B}$ within a heart rate response group illustrate the heart rate change waveforms for the CS + and $C S-$ trials. The right panels show the averaged heart rate change values across three time intervals of stimulus presentation $(0-1.3 \mathrm{~s}, 1.3-2.6 \mathrm{~s}, 2.6-3.9 \mathrm{~s})$. The lime point 0 s represents stimulus onset. Er ror bars indicate standard crrors. 


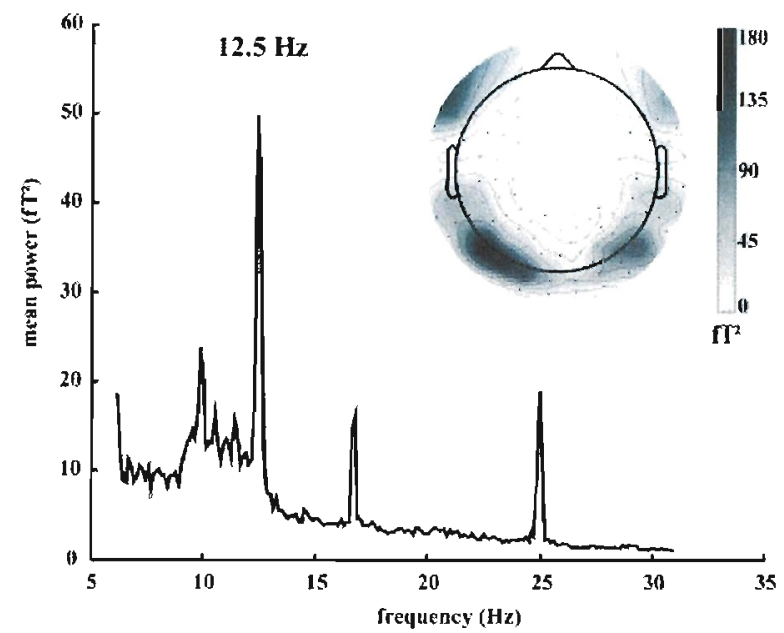

B

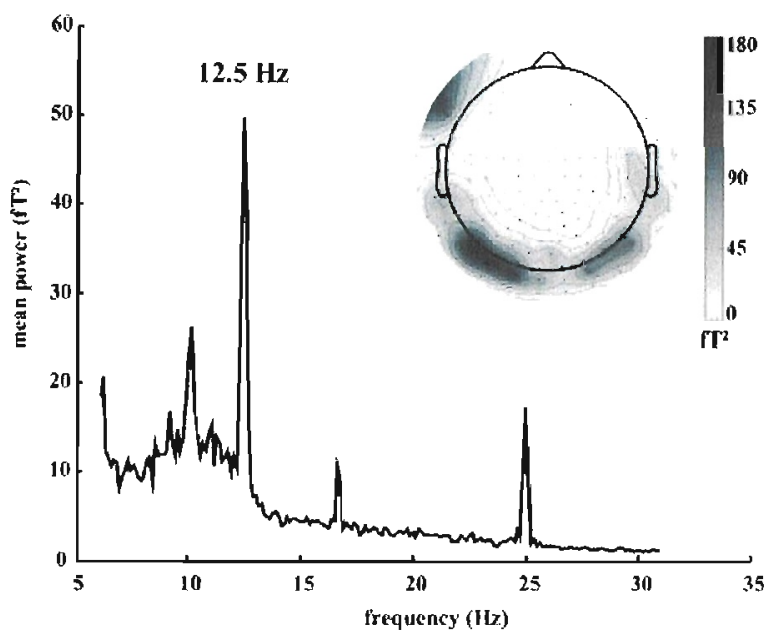

Acquisition

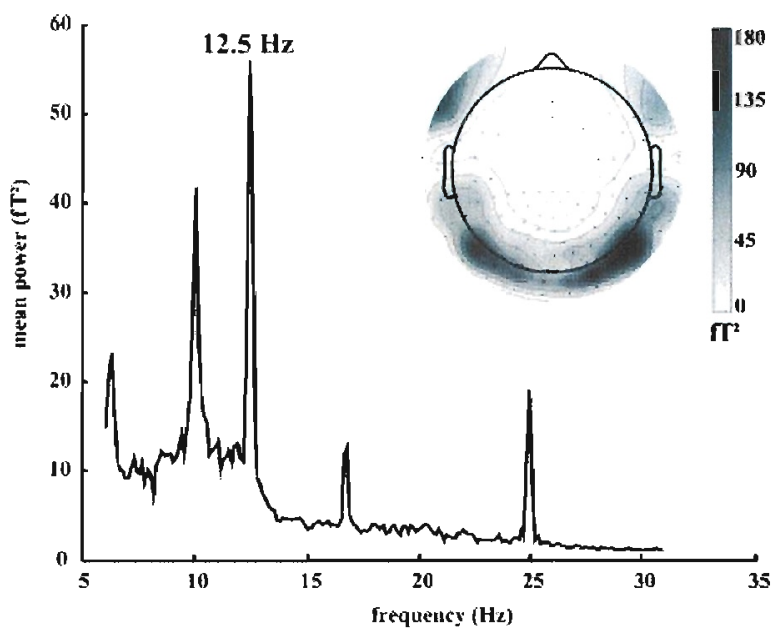

Extinction

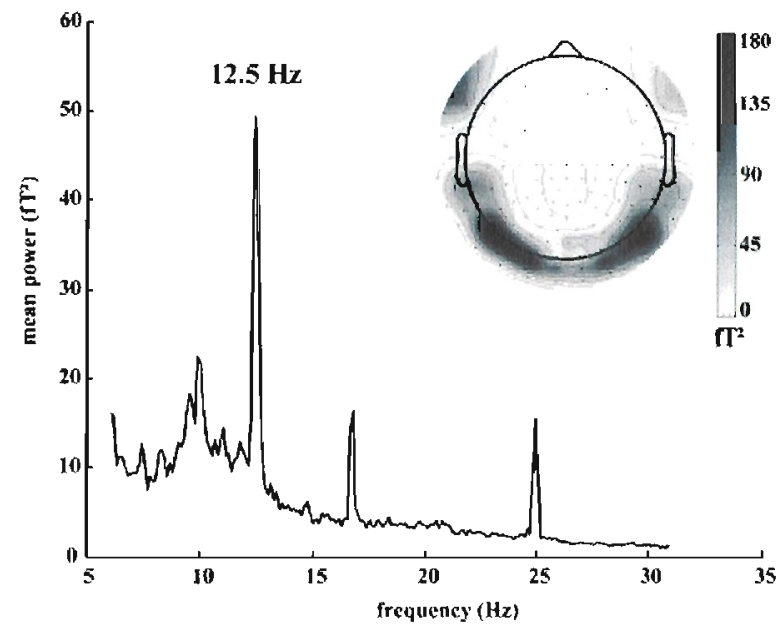

Figure 2. Mean power spectra across all MEG sensors, the entire CS + and CS - presentation time, and all subjects for acquisition (A) and cxtinction (B). A clcar peak at the $12.5-\mathrm{Hz}$ stimulation frequency is visible. The peak around $16 \mathrm{~Hz}$ is noisc from a nearby rail track. The $25-\mathrm{Hz}$ pcak represents the first harmonic of the $12.5-\mathrm{Hz}$ response. The inlays show MEG topographics of the $12.5-\mathrm{Hz}$ power estimation with a dipolar pattern over occipilal sensors. Gray scale bars indicate the power valucs at each sensor; sensors are shown as dots.

linear trend could be fitted to the heart rate response only for $\mathrm{CS}+$, indicating a stronger slowing of heart rate to the reinforced stimulus (linear time $\times$ condition: $F[1,40]=4.2, p<.05$; linear time during $\mathrm{CS}+: F[1,20]=18.1, p<.001$; linear time during CS- : n.s.; see Figure $1 \mathrm{~B}$ ).

\section{FFT Power Values}

Figure 2 depicts the mean power values and the corresponding ssVEF MEG topographies obtained during CS+ and CS presentation in acquisition and extinction blocks. During acquisition trials and extinction trials, there were no differences in 12.5- $\mathrm{Hz}$ ssVEF power values estimated across the entire presentation time of conditioned stimuli and all subjects (acquisition: $\mathrm{CS}+127.5 \mathrm{fT}^{2} \pm 32.5 \mathrm{fT}^{2} ; \mathrm{CS}-137.7 \mathrm{fT}^{2} \pm 32.4 \mathrm{fT}^{2} ;$ $t[19]=-1.0, p=.3$; extinction: $\mathrm{CS}+125.7 \mathrm{fT}^{2} \pm 35.4 \mathrm{fT}^{2}$;
$\left.\mathrm{CS}-143.0 \mathrm{fT}^{2} \pm 50.0 \mathrm{fT}^{2} ; \iota[19]=-1.2, p=.25\right)$. The pronounced $12.5-\mathrm{Hz}$ peak and the MEG topographies indicating occipital and parietal sources suggest satisfying signal-to-noise ratio even with the low trial count, which is in line with previous work using steady-state signals (e.g., Müller et al., 1997). Note that with magnetometer systems sources are assumed to lie between maxima in power plots.

\section{Minimum Norm Estimation Data}

Accelerators. During habituation trials, statistical mapping did not indicate any differential stimulus-driven cortical activation in heart rate accelerators for $\mathrm{CS}+$ and $\mathrm{CS}-$. However, during acquisition heart rate accelerators exhibited greater ssVEF in visual and parietal brain regions (see Figure 3) 
A
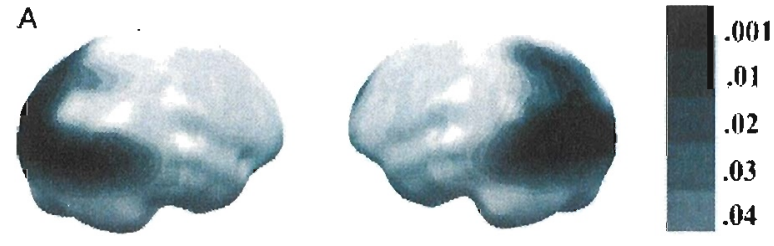

$p$ value

B
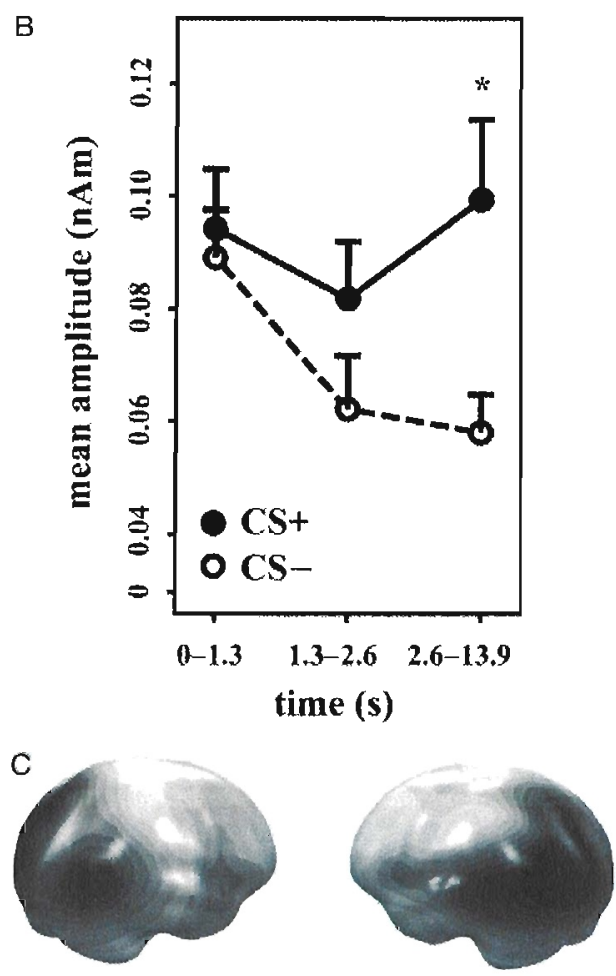

$2.6-3.9$

time interval (s)

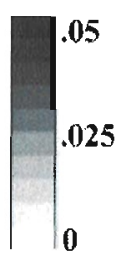

nAm

Figure 3. A: Differential ss VEF responses to the $\mathrm{CS}+$ and $\mathrm{CS}-$ in heart ratc accelerators localized with the minimum norm estimation technique. Statistical mapping of the interaction of condition (CS+, CS - ) and time $(0-1.3 \mathrm{~s}, 1.3-2.6 \mathrm{~s}, 2.6-3.9 \mathrm{~s})$ revealed significant sourcc clusters in visual and parictal cortex. The grayscale bar indicates corrected $p$ valucs. B: Mean amplitudes across significant source clusters were grcaler during late stimulus presentation time just before US onset for the CS +. Error bars indicate standard crrors. C: Difference map between minimum norm topographies for CSt and CS - trials during the late slimulus presentation interval shows greater activation in occipital and parictal cortex for the CS + . The grayscale bar indicales difference amplitudes

during the third time interval of stimulus presentation (2.6-3.9 s) for $\mathrm{CS}+$ than for $\mathrm{CS}-$ (comparison using mean amplitudes across significant occipital and parietal source clusters: $t[8]=3.4$, corrected $p<.05$; see Figure 3). During extinction no effects of condition (CS+, $\mathrm{CS}-$ ) were observed.

Decelerators. During habituation, acquisition, and extinction trials, statistical mapping did not reveal any source clusters that survived the false discovery rate procedure and met the spatial criterion (see Methods)

A
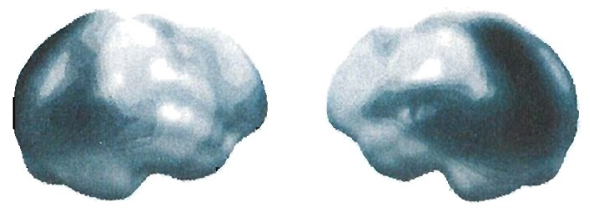

0.46
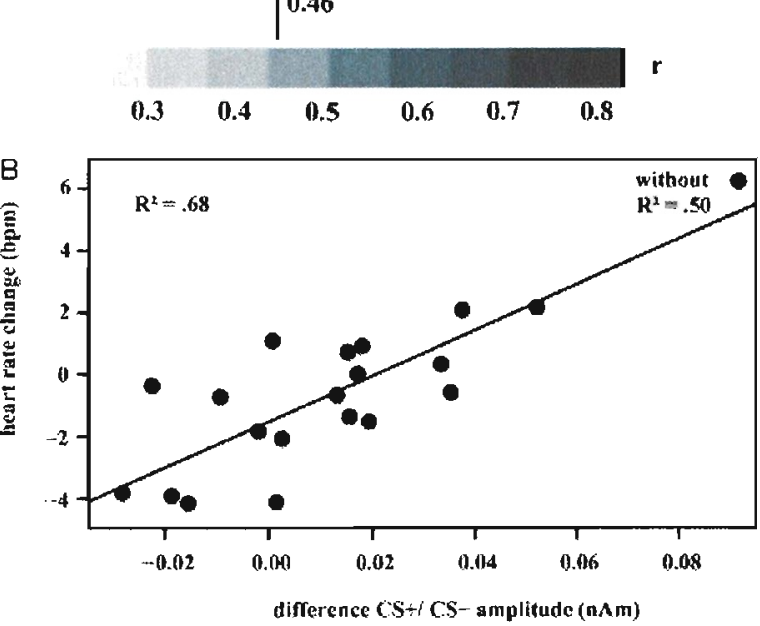

Figure 4. A: Distribution of correlation coefficients for the rclationship between heart rate change values and the difference in cortical activation during $\mathrm{CS}+$ and $\mathrm{CS}-$ presentation in late lime intervals during acquisition. The grayscale indicates the correlation coefficients. Coefficients greater than .46 were associated with corrected $p$ values less than .05. B: Regression analysis of the mean difference amplitude (CS+ minus CS - ) across source clusters of significant correlation and heart rate change values indicales a positive relationship, $F(1,18)=39.0$, $p<.000$ l, between the two measures. $R^{2}$ valucs are depicted using all subjects and discarding results of 1 participant with highest hcart rate change values and $\mathrm{CS}+/ \mathrm{CS}-$ amplitude difference.

\section{Correlational Analysis of Minimum Norm Estimation Data and} Heart Rate Change

To validate the above reported results of enhanced ssVEF in visual and parietal brain regions in heart rate accelerators during acquisition trials, a correlational analysis was undertaken using the entire sample $(N=20)$. The minimum norm difference scores at 273 dipole locations between $\mathrm{CS}+$ and $\mathrm{CS}-$ conditions during the third time interval of CS presentation $(2.6-3.9 \mathrm{~s}$; see Figure 3 ), in which accelerators differentiated $\mathrm{CS}+$ and CS - , were correlated with heart rate change during the second time window (1.3-2.6 s; see Figure 1), when accelerators differentiated CS+ and $\mathrm{CS}-$. Figure 4 shows the spatial distribution of the correlation coefficients. Its associated $p$ values were false discovery rate corrected, and only positive correlation coefficients exceeded the $p$ value threshold of $.05(r \geq .46)$. Positive correlation coefficients indicate that greater heart rate change values in CS+ trials during acquisition were associated with greater stimulusdriven sSVEF responses to the CS + compared to the $\mathrm{CS}-$ in visual and parietal brain regions.

Regression analysis using the mean difference between CS+ and CS - ssVEF amplitudes, averaged across the occipital and parietal sources identified by the mapping of the correlation coefficients, to predict heart rate confirmed the relationship between an accelerative heart rate component and greater 
stimulus-driven CS+ activation, $F(1,18)=39.0, \quad p<.0001$; $R^{2}=.68$ (see Figure 4). Even after removing the subject with greatest heart rate change values and $\mathrm{CS}+/ \mathrm{CS}-$ amplitude difference (see Figure 4, upper right corner of panel B), the regression analysis still indicated a strong relationship between the two measures, $F(1,17)=16.7, p<.001 ; R^{2}=.50$. The complementary regression analysis using the difference score $\mathrm{A} 1$ (see Methods) to account for realtive accelaration revealed the same relationship between enhanced $\mathrm{CS}+$ proccessing and an accelerative heart rate component, $F(1,18)=31.3, p<.0001 ; R^{2}=.65$.

\section{Discussion}

The aim of the present study was to investigate whether CS-US contingency awareness is a sufficient prerequisite for the acquisition of activity modulation in visual cortex by fear-relevant stimuli. Thus, at issue was whether subjects would show greater ssVEF activity in cortical sensory and attention networks only because of directed attention induced by instructing them about CS-US contingencies (expectancy) versus whether activity modulations in these networks depended on motivated attention (Lang et al., 1997) to fear-relevant stimuli, evidenced by relative heart rate acceleration. It should be noted here that in a triphasic heart rate response, an accelerative heart rate component can occur below baseline levels (see, e.g., Bradley et al., 200J; Kuniecki, Coenen, \& Kaiser, 2002).

FFT power values in the sensor space estimated across the entire poststimulus time interval did not differ between $C S+$ and $\mathrm{CS}-$ conditions in acquisition and extinction trials. This was expected based on earlier findings of differential ssVEF activity specifically in time intervals at the end of $\mathrm{CS}+/ \mathrm{CS}-$ presentation and only in heart rate accelerators (Moratti \& Keil, 2005). The overall FFT power analysis served to estimate the signal-tonoise level inherent in our data by examining the $12.5-\mathrm{Hz}$ spectral peak and its topographical distribution (see Figure 2). To test our present hypotheses regarding the time course and amplitude of facilitated visual cortical processing during $\mathrm{CS}+$ anticipation, we used the time-varying amplitude in source space.

During acquisition trials, late amplification in occipital and parietal brain regions when viewing the CS + was observed only in participants with an accelerative heart rate component. Heart rate decelerators did not show enhanced cortical activation to the $\mathrm{CS}+$ compared to the CS - . Statistical mapping of the relationship between heart rate change values and the difference between ssVEF amplitudes elicited by CS + versus CS - during acquisition across the entire sample of participants corroborated the different cortical response patterns in occipital and parietal cortical networks of heart rate accelerators and decelerators across the whole sample.

The accelerative heart rate components of 7 out of 9 heart rate accelerators exceeded the zero baseline (see Figure 4). The accelerative component of 2 heart rate accelerators was embedded in a general decelerative response pattern and did not reach the baseline level. This may be due to the target detection task used, as this kind of task may trigger a general OR response resulting in a decelerative heart rate response pattern. Furthermore, using auditory white noise as US in the present study may be less aversive than an electric shock, which has often been used in other conditioning studies. This also could have resulted in a smaller accelerative component of the heart rate response. In a previous fear conditioning study without initial information about contingencies, the accelerative heart rate component within a triphasic response pattern was smaller than in the current study, indicating that awareness may be related to stronger heart rate acceleration (see Moratti \& Keil, 2005, Figure 1).

Although 2 of the 9 heart rate accelerators did not push their accelerative heart rate component above baseline, the scatter plot of Figure 4 clearly indicates a relationship between heart rate change values and differentiated cortical activation in occipital and parietal brain regions with respect to the CS+. After discarding the responses of the participant with the highest heart rate acceleration, still $50 \%$ of the variance in ssVEF amplitude difference was predicted by heart rate acceleration. Further, using the difference between the fastest heart rate change of the second and the slowest heart rate change of the first time window (Al component) instead of absolute heart rate values for regression analysis revealed the same relationship between heart rate acceleration and enhanced CS + activation in occipital and parietal cortex.

Given these results, we argue that enhancement of ssVEF amplitudes when viewing the CS + was not driven by directed attention guided by expectancy alone, as has been reported in the field of spatial selective attention (Hillyard et al., 1997; Morgan et al., 1996; Müller \& Hillyard, 2000; Müller \& Hübner, 2002), but was associated with activation of the fear system as indexed by an accelerative heart rate component.

For heart rate accelerators, the involvement of occipital and parietal brain areas in fear conditioning parallels results from hemodynamic neuroimaging studies using fMRI and PET (Büchel et al., 1998; Cheng et al., 2003; Fischer et al., 2002; Fredrikson et al., 1995; Hugdahl, 1998; Knight et al., 2004; LaBar et al., 1998) demonstrating the engagement of visual sensory and cortical attention networks. This is in line with recent notions (Keil et al., 2005; Moratti et al., 2004) that motivationally significant stimuli modulate activity in attention systems. The present study also replicates previous results from our group suggesting that peripheral markers of fear may predict the presence of visual facilitation of the conditioned fear stimulus (Moratti \& Keil, 2005). In this previous study, however, we observed differential processing of the $\mathrm{CS}+$ for the heart rate accelerators after learning whereas in the present work, cortical facilitation for the CS+ occurred already during acquisition trials. This may suggest that in the previous, mostly unaware sample, learning was slower than in the present sample, which was fully informed about CS-US contingencies. In addition, the topographical distribution of enhanced ssVEF amplitude for the CS+ compromised larger occipital and parietal regions than in the previous study, suggesting that awareness in participants showing an accelerative heart rate component produced greater and more reliable effects.

Nevertheless, in both studies greater neuromagnetic responses to $\mathrm{CS}+$ depiction were restricted to subjects activating their fear system as indexed by accelerative heart rate components. Likewise, in both studies, facilitation of CS+ processing was observed in late time windows preceding US presentation. This facilitation of visual processing may have resulted from top-down processes exerted by fronto-parietal cortical networks, which is consistent with the source configuration observed here.

Our source estimation algorithm, however, did not account for deep structures such as the amygdaloid complex, a core region for Pavlovian fear conditioning (Davis, 1997; LeDoux, 2000). As a consequence, involvement of evolutionary old, deep 
structures cannot be ruled out and is indeed likely. As the amygdala is highly interconnected with visual cortex including the ventral stream (Amaral, 1986), the ssVEF amplitude enhancement in CS+ trials may reflect afferent input originating from amygdaloid complex. It is also possible that the amygdala facilitates sensory processing in early acquisition trials and that cortical attention systems adopt this role as soon as associations have been established. Consistent with this notion, hemodynamic neuroimaging studies have demonstrated a reduction in amygdala activation over trials in Pavlovian fear conditioning (Büchel et al., 1998; LaBar et al., 1998).

Converging evidence comes from the time course of magnetocortical facilitation for conditioned fear stimuli observed in the present study. Steady-state VEF responses in heart rate accelerators were enhanced in the late time interval (2.6-3.9 s), immediately before US presentation. This temporal pattern points to allocation of attentional resources to the visual modality, in the specific time window crucial for predicting the US. This concurs with previous notions that ssVEPs/ssVEFs represent reentrant activity modulation in sensory systems, indicating changes in widespread neural mass activity (Silberstein et al., 1990).

The results of the present study suggest that peripheral indicators of fear are important predictors of visual facilitation, although heart rate acceleration and enhanced ssVEF responses occurred at different latencies. Given these different time scales, a model assuming that heart rate acceleration leads to cortical facilitation seems too simplified. Here, we consider the accelerative heart rate component as a marker of a fear response, which indicates that a stimulus has acquired motivational relevance. Stimuli evoking fear responses have been hypothesized to be represented as neural networks, being associated with a number of complex physiological and behavioral processes that may partly occur in a parallel manner (Lang et al., 1997). Among other peripheral and central changes, associating the CS+ with fear responses is related to cortical facilitation specifically for the $\mathrm{CS}+$ during relevant time intervals.

The present work indicates that enhanced processing of the $\mathrm{CS}+$ in occipital and parietal brain regions is not merely due to instructed attention induced by known CS-US contingencies (i.e., by expectancy) but is driven by fear-related motivated attention due to activation of the defense system. Although the study did not measure expectancy of the US directly, it appeared that all subjects were well aware of the CS-US relationships because (1) they were told the contingencies, (2) the CS+ was reinforced $100 \%$ during acquisition, and (3) all subjects correctly identified the CS+ at the end of the experiment.

Present results oppose the proposals of Lacey and Lacey (1980) (1) that heart rate deceleration is related to increased intake of the stimulus, representing an OR; and (2) that heart rate acceleration is associated with stimulus rejection. According to this view, heart rate decelerators (rather than heart rate accelerators) should have shown increased ssVEFs to the CS+. Furthermore, an amplitude enhancement in occipital and parietal brain regions as a result of reflexive orienting would have been expected in early time intervals during presence of the CS+ (Graham, 1992; Lacey \& Lacey, 1980). In contrast, neuromagnetic sSVEF responses were enhanced in the late time interval (2.6-3.9 s) of CS+ presentation specifically in heart rate accelerators. This fine-tuned facilitation process focused on the time in which the US could have been expected suggests a timed activation of the motive systems driving attentional resources rather than a reflexive $O R$ in those subjects who exhibited a fear response. In turn, the enhanced cortical processing in heart rate accelerators could be reconciled with the Laceys' view if enhanced processing of a fear-relevant stimulus prevents distraction by irrelevant environmental stimuli (Lacey \& Lacey, 1980) in time periods critical for US detection. However, as we have collapsed data of all subjects decelerating their heart rate to the $\mathrm{CS}+$ to form the decelerators group and as our groups were not divided into moderate and strong heart rate decelerators as in previous studies (e.g., Hodes et al., 1985), effects for strong heart rate decelerators may have been concealed.

The present study demonstrates that the amplification of ssVEF responses in parietal and occipital brain areas during the $\mathrm{CS}+$ reflects fear-specific facilitation of the CS+ in aware subjects. Its time course and relationship with cardiac data suggest that during acquisition of a fear response, visual attention is allocated during time segments critical for US detection as a function of activation in the fear system, supporting the concept of motivated attention (e.g., Lang et al., 1997). Awareness of CS-US contingencies is not a sufficient prerequisite for fear conditioning with respect to enhanced cortical processing of the $\mathrm{CS}+$, although awareness seems to expedite learning and seems to produce stronger responses. In terms of the two-level account for fear conditioning in humans (Hamm \& Weike, 2005), learning under which circumstances a threatening event occurs (second level) is not sufficient to generate cortical facilitation for the $\mathrm{CS}+$, as subjects without an activation of the fear system (first level) did not show such an effect.

\section{REFERENCES}

Amaral, D. G. (1986). Amygdalohippocampal and amygdalocortical projeclions in the primalc brain. Advances in Experimental Medicine and Biology, 203, 3-17.

Armony, J. L., \& Dolan, R. J. (2001). Modulation of auditory neural responses by a visual context in human fear conditioning. Neuroreport, 12, 3407-3411.

Bagiclla, E., Sloan, R. P., \& Hcitjan, D. F. (2000). Mixcd-effects modcls in psychophysiology. Psychophysiology, 37, 13-20.

Bcnjamini, Y., \& Hochberg, Y. (1995). Controlling the false discovery ratc: A practical and powerful approach to multiple testing. Journal of the Royal Statistical Society Series B, 57 , 289-300.

Berg, P., \& Scherg, M. (1994). A multiple source approach to the correction of cye artifacts. Electroencephalography and Clinical Neurophysiology, 90, 229-24l.
Bradley, M. M., Codispoti, M., Cuthbert, B. N., \& Lang, P. J. (200I) Emotion and motivalion I: Defensive and appctitive reaclions in picture processing. Emotion, l, 276-298.

Bradlcy, M. M., Sabatinclli, D., Lang, P. J., Fitzsimmons, J. R., King, W., \& Desai, P. (2003). Activation of the visual cortex in motivated attention. Behavioral Neuroscience, 117, 369-380.

Büchcl, C., Morris, J., Dolan, R. J., \& Friston, K. J. (1998). Brain systems mediating aversive condilioning: An event-related fMRI sludy. Neuron, 20,947-957.

Cheng, D. T., Knight, D. C., Smith, C. N., Stein, E. A., \& Helmstetter, F. J. (2003). Functional MRI of human amygdala activity during Pavlovian fear conditioning: Stimulus proccssing versus response cxpression. Behavioral Neuroscience, $117,3-10$.

Davis, M. (1997). Neurobiology of fear responses: The rolc of the amygdala Journal of Neuropsychiatry and Clinical Neuroscience, 9, 382-402. 
Esteves, F., Parra, C., Dimberg, U., \& Öhman, A. (1994). Nonconscious associalive learning. Pavlovian conditioning of skin conductance responses to masked fear-relevant facial slimuli. Psychophysiology, 3I, 375-385.

Fernandez-Duque, D., \& Posner, M. I. (2001). Brain imaging of attentional networks in normal and pathological states. Journal of Clinical and Experimental Neuropsychology, 23, 74-93.

Fischer, H., Andersson, J. L., Furmark, T., Wik, G., \& Fredrikson, M. (2002). Right-sided human prefrontal brain activation during acquisition of conditioned fear. Emotion, 2, 233-24I.

Fredrikson, M., Wik, G., Fischer, H., \& Andersson, J. (1995). Affective and attentive neural networks in humans: A PET study of Pavlovian conditioning. Neuro Report, 7, 97-101.

Graham, F. K. (1992). Altention: The heartbeat, the blink, and the brain In B. A. Campell, H. Haync, \& R. Richardson (Eds.), Attention and information processing in infants and adults (pp. 3-29). Hillsdale, NJ: Erlbaum.

Hamm, A. O., \& Vaitl, D. (1996). Affeclive learning: Awareness and aversion. Psychophysiology, 33, 698-710.

Hamm, A. O., \& Weike, A. I. (2005). The neuropsychology of fear learning and fear regulation. International Joumal of Psychophysiology, 57, 5-14.

Hamm, A. O., Weike, A. I., Schupp, H. T., Treig, T., Dressel, A., \& Kessler, C. (2003). Affective blindsight: Intact fear conditioning to a visual cue in a cortically blind patient. Brain, I26, 267-275.

Hansen, P. C., \& O'Leary, D. P. (1993). The use of the L-curve in the regularization of discrele ill-posed problems. SIAM Journal of Scientific Compuring, 14, 1487-J 503.

Harc, R. D., \& Blevings, G. (1975). Defensive responses to phobic slimuli. Biological Psychology, 3, 1-13

Hauk, O., Keil, A., Elbert, T., \& Müller, M. M. (2002). Comparison of data transformation procedures to enhance topographical accuracy in lime series analysis of the human EEG. Journal of Neuroscience Methods, 113, 111-122.

Hillyard, S. A., Hinrichs, H., Tempelmann, C., Morgan, S. T., Hansen, J. C., Scheich, H., \& Hcinzc, H. J. (1997). Combining steady-state visual evoked potentials and $\mathrm{FMRI}$ to localize brain activity during sclective attention. Human Brain Mapping, 5, 287-292.

Hodes, R. L., Cook, E. W. 3rd, \& Lang, P. J. (1985). Individual differences in autonomic response: Conditioned association or condilioned fear? Psychophysiology, 22, 545-560.

Holm, S. (1979). A simplc sequentially rejective mul tiple test procedure. Scandinavian Journal of Statistics, 6, 65-70.

Hugdahl, K. (1998). Corfical control of human classical conditioning: Autonomic and positron cmission tomography data. Psychophysiotogy, 35, 170-178.

Jensen, O., \& Vanni, S. (2002). A new method to identify multiple sources of oscillatory activity from magnetocncephalographic data. Neurolmage, 15, 568-574

Junghöfer, M., \& Peyk, P. (2004). Analyse und Visualisierung von Hirnstrom- und Hirnmagnetfeld-Messungen. MATLAB Select, 2, $24-28$.

Kcil, A., Bradley, M. M., Hauk, O., Rockstroh, B., Elbert, T., \& Lang, P. J. (2002). Large-scale neural corrclates of affective piclure processing. Psychophysiology, 39, 641-649.

Kcil, A., Morati, S., Sabatinclli, D., Bradlcy, M. M., \& Lang, P. J. (2005). Additive effects of cmotional conlent and spatial selcctivc attention on electrocortical facilitation. Cerebral Cortex, 15, $1187-1197$.

Kcmp, A. H., Gray, M. A., Eide, P., Silberstcin, R. B., \& Nathan, P. J. (2002). Steady-statc visually cvoked potential topography during processing of emotional valence in healthy subjects. Neurolmage, 17 , $1684-1692$

Knight, D. C., Cheng, D. T., Smith, C. N., Stcin, E. A., \& Helmstetter, F. J. (2004). Neural substrates mcdiating human delay and trace fear conditioning. Jounnal of Neuroscience, 24, 218-228.

Knight, D. C., Nguyen, H. T., \& Bandeltini, P. A. (2003). Expression of conditional fear with and withoul awareness. Proceedings of the National Academy of Sciences, USA, 100, 15280-15283.

Kuniecki, M., Coenen, A. M., \& Kaiser, J. (2002). Correlation between long latency evoked potentials from amygdala and evoked cardiac response to fear conditioncd stimulus in rats. Acta Neurobiologiate Experimentalis, 62, 85-92.
LaBar, K. S., Gatenby, J. C., Gore, J. C., LeDoux, J. E., \& Phelps, E. A. (1998) Human amygdala activation during conditioned fear acquisition and extinction: A mixed-trial fMRI study. Neuron, 20, 937-945.

Lacey, B. C., \& Lacey, J. I. (1980). Presidential address, 1979. Cognitive modulation of time-dependent primary bradycardia. Psychophysiology, 17, 209-221.

Lang, P. J., Bradley, M. M., \& Cuthbert, B. N. (1997). Motivated attention: Affect, activation, and action. In P. J. Lang, R. F. Simons, \& M. T. Balaban (Eds.), Attention and orienting: Sensory and motivational processes (pp. 97-135). Hillsdale, NJ: Erlbaum

Lang, P. J., \& Hnatiow, M. (1962). Stimulus repetition and the heart rate response. Journal of Comparative and Physiological Psychology, 55, $781-785$.

LeDoux, J. E. (1993). Emotional networks in the brain. In J. M. H. Michael Lewis (Ed.), Handbook of emolions (pp. 109-118). New York: Guilford Press.

LeDoux, J. E. (2000). Emotion circuits in the brain. Annual Review of Netroscience, 23, 155-184.

Lipp, O. V., Sheridan, J., \& Siddlc, D. A. (1994). Human blink startlc during aversive and nonaversive Pavlovian conditioning. Joulnal of Experimental Psychologv, Animal Behavioral Processes, 20, 380-389.

Lovibond, P. F., \& Shanks, D. R. (2002). The role of awareness in Pavlovian conditioning: Empirical cvidence and theoretical implications. Journal of Experimental Psychology, Animal Behavioral Processes, 28, 3-26.

Moratli, S., \& Kcil, A. (2005). Cortical activation during Pavlovian fear conditioning depends on hearl rate response patterns: An MEG study. Brain Research Cognitive Brain Research, 25, 459-47I.

Morati, S., Keil, A., \& Stolarova, M. (2004). Motivated attention in emotional picture processing is reflected by activity modulation in cortical altention networks. Neurolmage, 21, 954-964.

Morgan, S. T., Hansen, J. C., \& Hillyard, S. A. (1996). Selective attention to stimulus location modulates the steady-state visual evoked potential. Proceedings of the National Academy of Sciences, USA, 93, $4770-4774$

Morris, J. S., Öhman, A., \& Dolan, R. J. (1998). Conscious and unconscious emotional learning in the human amygdala. Nature, 393, 467-470.

Müller, M. M., \& Hillyard, S. (2000). Concurrent recording of steadystatc and transient event-related polentials as indices of visual-spatial selective attention. Clinical Neurophysiology, /11, 1544-1552.

Müller, M. M., \& Hübncr, R. (2002). Can the spollight of allention be shaped like a doughnut? Evidence from stcady-state visual evoked potcntials. Psychological Sciences, 13, 119-124.

Müller, M. M., Teder, W., \& Hillyard, S. A. (1997). Magneloencephalographic recording of sleady-statc visual evoked corlical activity. Brain Topography, 9, 163-168

Öhman, A., Flykt, A., \& Esteves, F. (200l). Emotion drives attention: Detecling the snake in the grass. Journal of Experimental Psychology, General, 130, 466-478.

Öhman, A., \& Mincka, S. (2001). Fears, phobias, and preparedness: Toward an cvolved modulc of fcar and fear learning. Psychological Review, 108, 483-522.

Oldfield, R. C. (1971). The assessment and analysis of handedness: The Edinbourgh Invcnlory. Newropsychologia, 9, 97-113.

Olsson, A., \& Phelps, E. A. (2004). Learned fear of "unscen" faces after Pavlovian, observational, and instructed fear. Psychological Science, $15,822-828$.

Pearce, J. M., \& Hall, G. (1980). A model for Pavlovian learning: Variations in the effectiveness of conditioned but nol of unconditioned stimuli. Psychological Review, 87, 532-552.

Perlstcin, W. M., Cole, M. A., Larson, M., Kclly, K., Scignourcl, P., \& Keil, A. (2003). Steady-state visual evoked potentials reveal frontallymediated working memory activity in humans. Neuroscience Letters, $342,191-195$

Pinhciro, J. C., \& Bales, D. M. (2000). Mixed-effects models in $S$ and $S$ PLUS (1si cd). New York, Bcrlin, Heidelberg: Springer.

Regan, D. (1989). Human brain electrophysiology: Evoked potentials and evoked magnetic fields in science and medicine. New York: Elsevier.

Rescorla, R. A. (1988). Pavlovian conditioning. It's nol what you think it is. The American Psychologist, 43, 151-160.

Schupp, H. T., Junghofer, M., Weike, A. I., \& Hamm, A. O. (2003) Emotional facilitation of sensory processing in the visual cortex. Psychological Science, 14, 7-13. 
Searle, S. R., Casella, G., \& McCulloch, C. E. (1992). Variance Components. New York: Wiley.

Shi, C., \& Davis, M. (2001). Visual pathways involved in fear conditioning measured with fear-potentiated startle: Behavioral and anatomic studies. Journal of Neuroscience, 21, 9844-9855.

Silberstein, R. B., Schier, M. A., Pipingas, A., Ciorciari, J., Wood, S. R. \& Simpson, D. G. (1990). Steady-state visually evoked potential topography associated with a visual vigilance task. Brain Topography, 3 , $337-347$.

Uusitalo, M. A.. \& Ilmoniemi, R. J. (1997). Signal-space projection method for separating MEG or EEG into components. Medical \& Biological Engineering \& Computing, 35, 135-140.
Wiens, S., Katkin, E. S., \& Öhman, A. (2003). Effects of trial order and differential conditioning on acquisition of differential shock expectancy and skin conductance conditioning to masked stimuli. Psychophysiology, 40, 989-997.

Wiens, S., \& Öhman, A. (2002). Unawareness is more than a chance event: Comment on Lovibond and Shanks (2002). Journal of Experimental Psychology, Animal Behavioral Processes, 28, 27-31.

Wilson, R. S. (1974). CARDIVAR: The statistical analysis of heart rate data. Psychophysiology, 11, 76-85. 